\title{
The molecular basis for allelic differences suggests Restorer-of-fertility 1 is a complex locus in sugar beet (Beta vulgaris L.)
}

Takumi Arakawa ${ }^{1,2}$, Muneyuki Matsunaga', Katsunori Matsui ${ }^{1}$, Kanna Itoh', Yosuke Kuroda ${ }^{3}$, Hiroaki Matsuhira', Kazuyoshi Kitazaki ${ }^{1}$ and Tomohiko Kubo ${ }^{1 *}$ (D)

\begin{abstract}
Background: Cytoplasmic male sterility (CMS) is a widely used trait for hybrid seed production in many crops. Sugar beet CMS is associated with a unique mitochondrial protein named preSATP6 that forms a 250-kDa complex. Restorer-of-fertility 1 (Rf 1 ) is a nuclear gene that suppresses CMS and is, hence, one of the targets of sugar beet breeding. Rf1 has dominant, semi-dominant and recessive alleles, suggesting that it may be a multi-allelic locus; however, the molecular basis for differences in genetic action is obscure. Molecular cloning of Rf1 revealed a gene (orf20) whose protein products produced in transgenics can bind with preSATP6 to generate a novel 200-kDa complex. The complex is also detected in fertility-restored anthers concomitant with a decrease in the amount of the 250-kDa complex. Molecular diversity of the Rf1 locus involves organizational diversity of a gene cluster composed of orf2O-like genes (RF-Oma1s). We examined the possibility that members of the clustered RF-Oma1 in this locus could be associated with fertility restoration.
\end{abstract}

Results: Six yet uncharacterized RF-Oma1s from dominant and recessive alleles were examined to determine whether they could generate the 200-kDa complex. Analyses of transgenic calli revealed that three RF-Oma1s from a dominant allele could generate the 200-kDa complex, suggesting that clustered RF-Oma1s in the dominant allele can participate in fertility restoration. None of the three copies from two recessive alleles was 200-kDa generative. The absence of this ability was confirmed by analyzing mitochondrial complexes in anthers of plants having these recessive alleles. Together with our previous data, we designed a set of PCR primers specific to the 200-kDa generative RF-Oma1s. The amount of mRNA measured by this primer set inversely correlated with the amount of the 250-kDa complex in anthers and positively correlated with the strength of the Rf1 alleles.

Conclusions: Fertility restoration by sugar beet Rf1 can involve multiple RF-Oma1s clustered in the locus, implying that stacking 200-kDa generative copies in the locus strengthens the efficacy, whereas the absence of 200-kDa generative copies in the locus makes the allele recessive irrespective of the copy number. We propose that sugar beet $R f 1$ is a complex locus.

Keywords: Cytoplasmic male sterility, Nuclear-mitochondrial interaction, Hybrid breeding, Oma1, Allelic diversity, Plant reproduction

\footnotetext{
* Correspondence: tomohiko@abs.agr.hokudai.ac.jp

'Research Faculty of Agriculture, Hokkaido University, Kita-9, Nishi-9, Kita-ku, Sapporo 060-8589, Japan

Full list of author information is available at the end of the article
}

(c) The Author(s). 2020 Open Access This article is licensed under a Creative Commons Attribution 4.0 International License, which permits use, sharing, adaptation, distribution and reproduction in any medium or format, as long as you give appropriate credit to the original author(s) and the source, provide a link to the Creative Commons licence, and indicate if changes were made. The images or other third party material in this article are included in the article's Creative Commons. licence, unless indicated otherwise in a credit line to the material. If material is not included in the article's Creative Commons licence and your intended use is not permitted by statutory regulation or exceeds the permitted use, you will need to obtain permission directly from the copyright holder. To view a copy of this licence, visit http://creativecommons.org/licenses/by/4.0/ The Creative Commons Public Domain Dedication waiver (http://creativecommons.org/publicdomain/zero/1.0/) applies to the data made available in this article, unless otherwise stated in a credit line to the data. 


\section{Background}

Cytoplasmic male sterility (CMS) is a mitochondrialencoded trait that is a prerequisite for hybrid seed production in some crop species [1-4]. CMS has often been associated with specific proteins in male-sterility inducing mitochondria, but the primary structures of these proteins differ among species [5, 6]. Some CMS-specific proteins, however, share several features such as having a hydrophobic domain [7]. In some crops such as maize, rapeseed with radish CMS, and sugar beet, CMS-specific proteins have been found in the mitochondrial membrane as oligomer forms [8-10].

CMS is suppressed by a nuclear gene termed Restorerof-fertility (Rf) [11]. Usually, a dominant $R f$ allele suppresses CMS expression. Hence, seed parents of hybrid seed production should be homozygous recessive. On the other hand, when the $F_{1}$ hybrid is meant for producing seed or fruit, the $F_{1}$ plant should be fertility restored to secure pollination. As such, a discriminating $R f$ genotype is quite important for hybrid breeding, but $R f$ and $r f$ plants are phenotypically indistinguishable when they are combined with non-sterility inducing mitochondria. Therefore, uncovering the molecular basis for allelic differences in $R f$ will be a great help toward advancing hybrid breeding.

Molecular cloning of $R f$ revealed that its gene product is variable, but the most prominent class encodes pentatrico peptide repeat (PPR) proteins that are involved in post-transcriptional processing of mitochondrial genes [12-14]. PPR genes constitute a large gene family in the plant genome, and PPR-type $R f$ belongs to a subclass termed $R f$-PPR-like genes $(R F L)$ [15]. PPR-type $R f$ tends to cluster with $R F L$ : the organization of the RFL-containing gene cluster is varied among genetic resources [1618]. The significance of such a variable gene cluster is, however, unclear. From the viewpoint of crop breeding, diagnosis of $R f$ alleles is important. An unanswered question at present is whether the dominant/recessive nature of an $R f$ allele depends solely on the presence/absence of a specific gene copy in the cluster; in other words, whether the rest of the $R f$-like gene copies can be ignored for allelic diagnosis.

Sugar beet cultivars are hybrids derived from the use of CMS [19]. Sugar beet CMS was discovered by Owen [20] and has been associated with a specific $39 \mathrm{kDa}$ mitochondrial protein that is encoded by an origin-unknown open reading frame (ORF), preSatp6 [10]. Translation product of preSatp6 can be found in all examined organs [10]. This protein is highly hydrophobic and is detected from a 250$\mathrm{kDa}$ protein complex when mitochondria are lysed in a mild detergent such as digitonin [21].

The genetics of fertility restoration in sugar beet is complex in some cases [22], but one of the best characterized sugar beet $R f \mathrm{~s}$ is $R f 1$ [23]. Molecular cloning of $R f 1$ revealed a gene cluster whose members resemble Oma1, a yeast gene involved in mitochondrial quality control [23]. The Oma1-like genes in the $R f 1$ locus (hereafter RF-Oma1) are non-canonical Oma1 genes because another apparently orthologous Oma1 gene exists in the sugar beet genome; RF-Oma1 has likely evolved by gene duplication followed by neofunctionalization [24].

In a transgenic experiment, one of the $R F-O m a 1$ genes was shown to increase pollen fertility [23]. When this RF-Oma1 copy is expressed in suspension cells of CMS sugar beet, its translation products can bind with preSATP6 protein to generate a novel $200-\mathrm{kDa}$ protein complex, whereas an RF-Oma1 from a recessive $r f 1$ allele has no such activity [21]. The 200-kDa complex was also detected in $R f 1$ fertility-restored anthers, indicating that the appearance of the $200-\mathrm{kDa}$ complex is a hallmark of molecular interaction between RF-Oma1 and preSatp6 [21]. Concomitant with the appearance of the 200-kDa complex in fertility-restored anthers, the amount of the $250-\mathrm{kDa}$ complex is highly reduced, yet the total amount of monomeric preSATP6 protein is almost unchanged [21]. We interpreted this phenomenon to be an alteration of the higher-order structure of preSATP6 protein by a molecular chaperone-like activity exerted by RF-Oma1 [21].

Our particular interest is the molecular diversity within the $R f 1$ locus. To date, we have determined the nucleotide sequences of six $R f 1 / r f 1$ alleles that differ in RF-Oma1 copy number (Fig. 1) (for amino acid sequence homologies among these genes, see Additional file 1: Table S1). One of these alleles is a semi-dominant NK$305 R f 1$ whose homozygote is fully fertile, whereas the heterozygote is semi-fertile [25]. This genetic action contrasts with a dominant NK-198 Rf1 whose heterozygote is fully fertile in the same condition, making us to infer that $R f 1$ alleles in beet genetic resources have diverged in functionally [25]. The molecular organization of $R f 1$ seems to have diverged significantly [26, 28, 29] with many of these alleles yet uncharacterized; hence, other alleles with different genetic actions are possible. Determining the molecular basis for differences in genetic action is necessary for evaluating genetic resources to find novel $R f 1$ alleles.

Before the present study, six out of eleven $R F$ Oma1 copies in Fig. 1 were uncharacterized (orf2 $O_{N K-219-1}$ is an apparent pseudogene). This lack of information prompted us to investigate $R f 1$ diversity by completely characterizing all of the RF-Oma1 genes in Fig. 1. To our surprise, the dominant NK$198 R f 1$ is composed of four $200-\mathrm{kDa}$ generative $R F$ Oma1 copies. This finding caused us to propose the possibility that a specific $R F-O m a 1$ copy may not be the determinant of the allele's nature, but rather the 


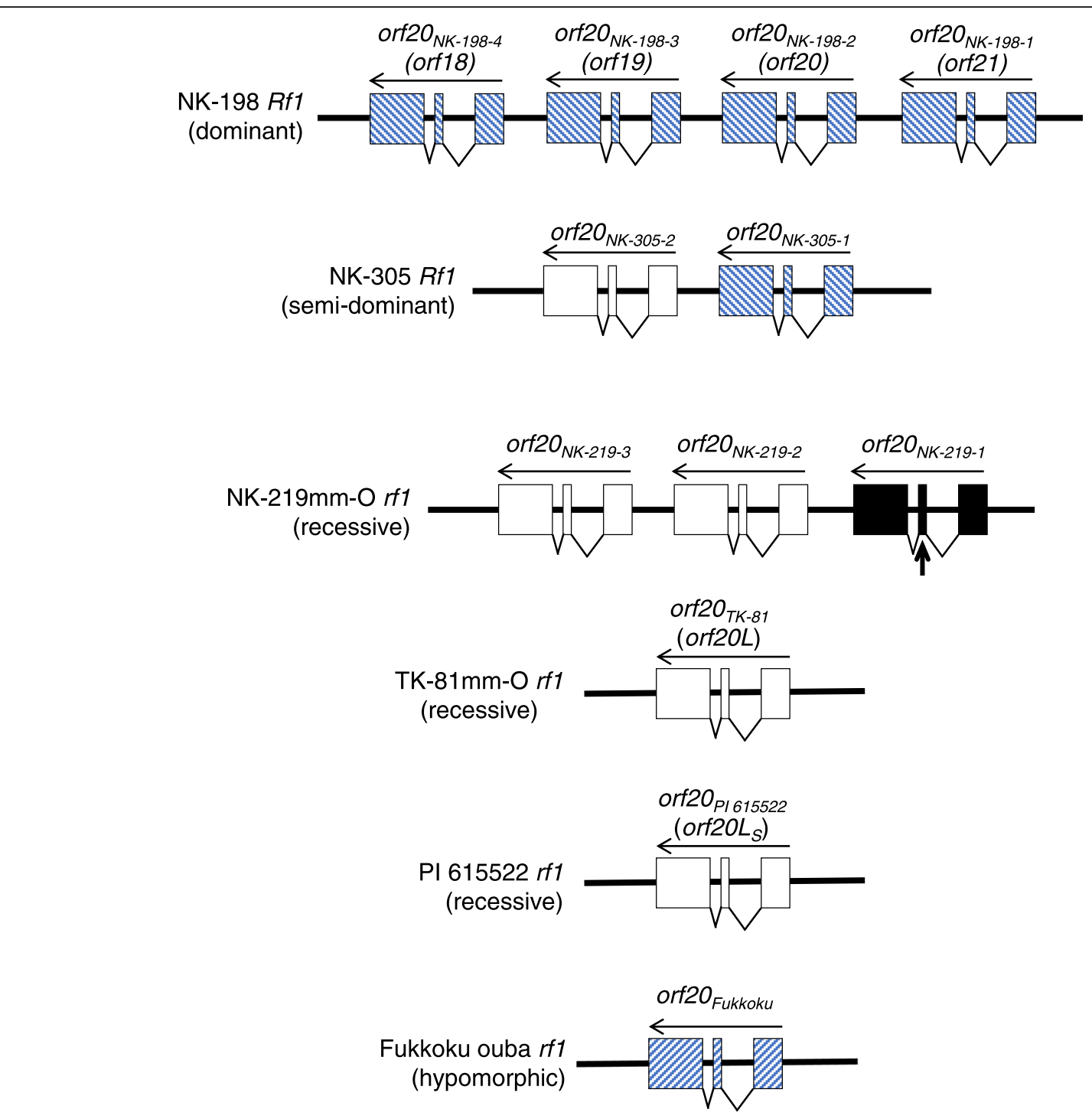

Fig. 1 Organizational summary of beet Rf1 loci. Names of alleles are shown at the left with their genetic action in parentheses. Boxes and wedges indicate exons and introns, respectively. Gene directions are from right to left. Names of individual RF-Omal are shown above the genes with the old names used in previous papers in parentheses. The 200-kDa generative copies are striped; non-generative copies are open; and a pseudogene is filled. A vertical arrow indicates the position of a premature stop codon. This study characterized orf20 $0_{N K-198-4,}$ orf20 $0_{N K-198-3,}$ $\operatorname{orf2}_{N K-198-1}$, orf20 $0_{N K-219-3}$, orf20 $0_{N K-219-2}$ and orf20 1 615522. Sources of other information about these loci are [23, 25-27]

total amount of mRNA associated with the generation of the $200-\mathrm{kDa}$ complex is one of the keys for defining the strength of the allele. In fact, we found an inverse correlation between the amount of mRNA and the amount of the $250-\mathrm{kDa}$ complex. The amount of mRNA well explains the difference in the allele's strength. Our finding implies that an increase in the number of $200-\mathrm{kDa}$ generative RF-Oma1 copies strengthens the allele, whereas non-generative $R F$ Oma1 copies provide nothing to the allele for fertility restoration. We propose that sugar beet $R f 1$ may be a complex locus whose action is determined by clustered RF-Oma1 copies.

\section{Results}

A dominant $R f 1$ allele decreases the accumulation of the 250-kDa protein complex in a gene-dose dependent

\section{manner}

During the course of our genetic analysis, we examined whether a dominant NK-198 Rf1 allele had a genedosage effect on the amount of the $250-\mathrm{kDa}$ protein complex. We used a $\mathrm{BC}_{2} \mathrm{~F}_{2}$ population derived from a cross between TA-33BB-CMS (a CMS line) and NK-198 (the donor line of NK-198 Rf1) to select homozygotes and heterozygotes of NK-198 Rf1. Although both genotypes were phenotypically indistinguishable as they were fully fertile in our greenhouse [25], it was possible to 
diagnose the $R f 1$ genotype by using the s17 DNA marker [25]. NK-198 Rf1 is identified by a specific PCR band pattern named $\mathrm{p} 1$, whereas the $r f 1$ from TA-33BB-CMS (the same as TK-81 mm-O rff in Fig. 1) is identified by p4. We selected homozygotes and heterozygotes of NK$198 R f 1$ and collected their immature anthers. Total anther cellular proteins were prepared in a buffer containing digitonin and electrophoresed on Blue Native (BN) polyacrylamide gels to detect protein complexes. Immunoblot analysis with anti-preSATP6 used conditions where the signal intensity of the $250-\mathrm{kDa}$ complex could be quantified; sample amount, concentration of primary antisera and exposure time were adjusted as reported in [25]. Compared to the $250-\mathrm{kDa}$ signal bands of $r f 1 r f 1$ from the same population, those of the heterozygotes were highly reduced but the faint bands could be seen (Fig. 2a); whereas, the $250-\mathrm{kDa}$ signal bands of the homozygotes were almost invisible (Fig. 2a). It is unlikely that this decrease in the $250-\mathrm{kDa}$ complex was due to an insufficient amount of loaded sample or inappropriate sample preparation because the levels of another mitochondrial complex detected by anti-COXI were comparable among the samples (Fig. 2b). These results suggested that NK-198 Rf1 exerts a gene-dosage effect on the accumulation of the 250-kDa complex, although we were unaware of such a cumulative effect on the phenotype.

\section{All RF-Oma1 copies in NK-198 Rf1 have the potential to generate 200-kDa complexes}

We further investigated the NK-198 Rf1 allele at the molecular level to determine how such a large genetic effect on fertility restoration was exerted. According to Matsuhira et al. [23], NK-198 Rf1 is comprised of four RF-Oma1 copies, orf18 to orf 21 (hereafter termed orf $2 O_{N K-198-4}$ to orf $20_{N K-198-1}$, respectively; see Fig. 1). Kitazaki et al. [21] showed that the translation products of orf $2 \mathrm{O}_{\mathrm{NK}-198-2}$

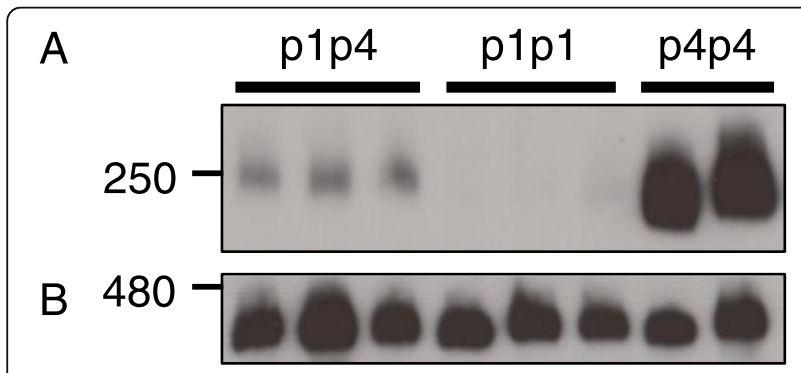

Fig. 2 Immunoblot analysis of total cellular proteins from immature anthers collected from a $\mathrm{BC}_{2} \mathrm{~F}_{2}$ population derived from TA-33BBCMS $\times$ NK-198. Protein samples were electrophoresed in Blue Native polyacrylamide gels (4-16\%). Size markers $(\mathrm{kDa})$ are shown on the left. DNA marker types are shown at the top; genotypes p1p4 and p1p1 are heterozygous and homozygous forms of NK-198 Rf1, respectively. Genotype p4p4 is a homozygous recessive (i.e. $r f 1$ f $f$ ). Immunoblots were probed with anti-preSATP6 (a) or anti-COXI (b) (formerly orf20) have the ability to bind to preSATP6 protein and to generate a unique $200-\mathrm{kDa}$ protein complex on BN polyacrylamide gels; however, the other RF-Oma1 copies remained uncharacterized. In this study, we investigated whether the three uncharacterized RF-Oma1 copies have the same potential for generating the $200-\mathrm{kDa}$ protein complex as orf $20_{N K-198-2}$. Each of the three copies (orf20 ${ }_{N K-198-1}$, orf2 $0_{N K-198-3}$, and orf $\left.20_{N K-198-4}\right)$ was fused to a FLAG tag and regulated by the Cauliflower Mosaic Virus (CaMV) $35 \mathrm{~S}$ promoter in binary vectors. The transgenes were introduced into CMS sugar beet suspension cells. Mitochondrial proteins from the transgenic cells were separated by BN-PAGE and probed with anti-preSATP6 to identify selectively the mitochondrial protein complex containing preSATP6. Smeared images were detected on the immunoblots, as was seen in previous studies (e.g. [25]). To our surprise, signal bands of $200-\mathrm{kDa}$ were seen in the lanes of all four samples of RF-Oma1 derived from NK-198 Rf1 (Fig. 3), suggesting that all four sequences produce a protein that can interact with preSATP6 protein.

Although Matsuhira et al. [23] suggested that all $R F$ Oma1 copies in NK-198 Rf1 were expressed, the relative transcript levels produced by the four sequences were unknown. The largest obstacle for quantifying transcript abundance was the high sequence similarity among the four RF-Oma1 copies, which precluded us from designing primer pairs to specifically quantify each of the four RF-Oma 1 mRNA species by PCR. Comparing the four RF-Oma1 sequences, we noticed small insertions/deletions (indels) and several single nucleotide polymorphisms (SNPs) that enabled us to infer the ratio of $R F$ Oma1 mRNA species (Additional file 2: Figure S1). We focused on a 6-bp indel in exon 1 and two SNPs in the 3 ' trailer (Additional file 2: Figure S1); the indel distin-

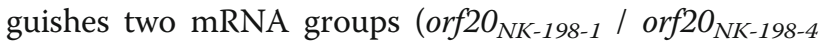
and orf $20_{N K-198-2} /$ orf $20_{N K-198-3}$ ), and the two SNPs distinguish between three mRNA groups $\left(\right.$ orf $20_{N K-198-4}$, orf $2 O_{N K-198-3}$, and orf $\left.20_{N K-198-1} / \operatorname{orf}_{2 O_{N K-198-2}}\right)$. Therefore, transcriptome data from anthers expressing NK198 Rf1 should contain reads of RF-Oma1 mRNA that can be divided into these groups.

A fertility-restored $\mathrm{BC}_{6} \mathrm{~F}_{1}$ plant derived from a cross between TA-33BB-CMS and NK-198 was selfed to obtain the NK-198 Rf1 homozygote, which the s17 marker should identify as p1p1. RNA samples were extracted from tetrad-stage anthers before we conducted RNA-seq analysis. Read counts are summarized in Additional file 3: Table S2. We estimated the ratios of transcripts derived from the four RF-Oma1 of NK-198 Rf1 (Table 1); for example, we obtained transcript ratios of groups such as orf $20_{N K-198-1} /$ orf $20_{N K-198-4}$. On the other hand, the ratio of orf $20_{N K-198-4}$ transcripts in the RF-Oma1 transcript pool of the NK-198 Rf1 homozygote was determined by 


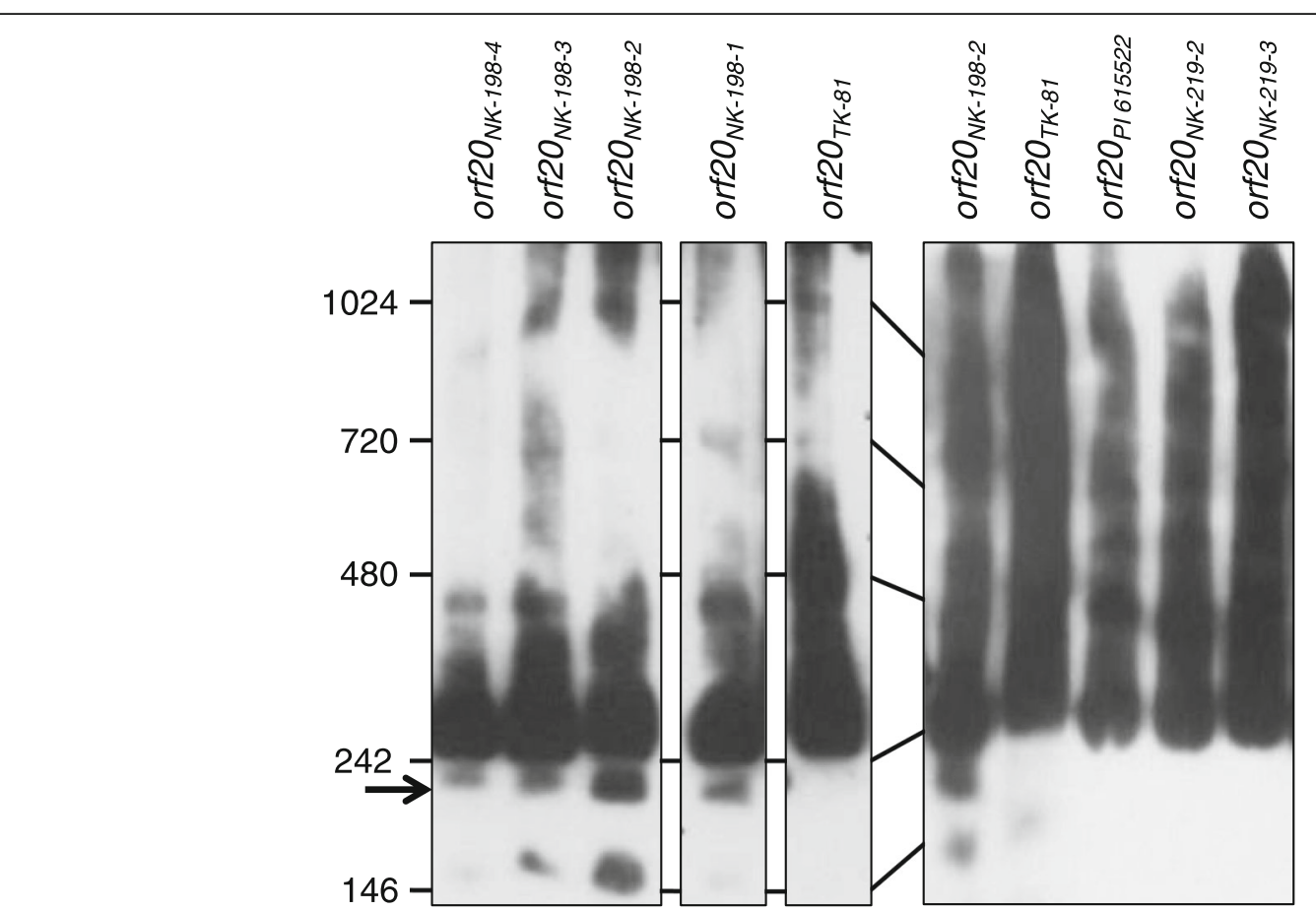

Fig. 3 Immunoblot analysis of crude mitochondrial proteins extracted from transgenic cell lines. Protein samples were electrophoresed in Blue Native polyacrylamide gels (4-16\%). Size markers $(\mathrm{kDa})$ are shown on the left. FLAG-fused construct names are shown at the top. An arrow indicates the location of a 200-kDa band that is the hallmark of post-translational interaction with preSATP6

utilizing SNPs in the 3' UTR (Table S2). Using these values, the ratio of orf $20_{N K-198-1}$ transcripts was calculated. Although the obtained values were approximations, the most abundant transcript was likely orf $20_{N K-198-2}$, followed by orf $20_{N K-198-4}$. The expression levels of the two lesser-expressed genes, orf $2 O_{N K-198-1}$ and orf $2 O_{N K-198-3}$, were comparable.

\section{Analysis of recessive alleles from different origins}

We also examined whether the remaining three uncharacterized RF-Oma1 copies (i.e. copies from NK-219 mmO rf1 and PI 615522 rf1) had the potential for generating the 200-kDa complex. In this study, we first selected two sugar beet lines, PI 518644 and PI 615522, that have orf $2 O_{N K-219-1}$ to orf $2 O_{N K-219-3}$ and orf $2 O_{P I} 615522$, respectively [26]. PI 518644 and PI 615522 are registered as 'O-

Table 1 Ratios of four RF-Omal mRNAs in anthers of NK-198 Rf1 homozygotes

\begin{tabular}{|c|c|c|c|c|}
\hline \multirow[t]{2}{*}{ RF-Oma1 } & \multicolumn{4}{|c|}{ Relative expression ratio } \\
\hline & $\overline{N \_1}{ }^{1}$ & $\mathrm{~N} \_2^{1}$ & N_3 ${ }^{1}$ & Mean $^{2}$ \\
\hline orf20 ${ }_{N K-198-1}$ & 0.20 & 0.17 & 0.16 & $0.18^{c}$ \\
\hline $\operatorname{orf}_{20}{ }_{N K-198-2}$ & 0.39 & 0.41 & 0.38 & $0.39^{a}$ \\
\hline $\operatorname{orf}_{20}{ }_{N K-198-3}$ & 0.16 & 0.17 & 0.17 & $0.17^{c}$ \\
\hline orf20 $0_{N K-198-4}$ & 0.25 & 0.25 & 0.29 & $0.26^{b}$ \\
\hline
\end{tabular}

${ }^{1}$ Biological replicates

${ }^{2}$ Differences in the letters indicate significance at $p<0.05$ using Tukey's multiple comparison test type', a specific genotype that lacks a restoring allele but has non-sterility inducing mitochondria. The two lines were crossed with TA-33BB-CMS. All $\mathrm{F}_{1}$ plants (sixteen from TA-33BB-CMS x PI 518644 and five from TA33BB-CMS x PI 615522) were completely male sterile.

Expression of RF-Oma1 in the $\mathrm{F}_{1}$ plants was examined by reverse transcription-quantitative PCR (RTqPCR). Total cellular RNAs were extracted from anthers at the meiotic and tetrad stages. RF-Omal mRNAs were simultaneously detected by the primers common to all the copies. The results are summarized in Table 2. Expression levels were generally higher at the meiotic stage than at the tetrad stage in every genotype. $F_{1}$ plants of TA-33BB-CMS $x$ PI 615522 expressed RF-Oma1 at a level comparable to that of TA-33BB-CMS, whereas those of TA-33BBCMS x PI 518644 were 1.8 to 2.6-times higher than that of TA-33BB-CMS (Table 2). The difference appeared to be associated with the copy number of $R F$ Oma1 in the zygote (Table 2).

We tested whether any of the RF-Oma1 translation products in PI 615522 or PI 518644 was $200-\mathrm{kDa}$ generative. Total cellular proteins of immature anthers collected from $F_{1}$ plants were subjected to BN-PAGE. Immunoblot analysis using anti-preSATP6 revealed that the two F1 plants gave images similar to that of TA33BB-CMS. No 200-kDa signal band was detected even after prolonged exposures (Fig. 4). 
Table 2 Relative transcript abundance of RF-Oma1 measured by RT-qPCR in male sterile plants with recessive rf1 alleles from different origins $(n=2)$

\begin{tabular}{|c|c|c|c|c|}
\hline \multirow{2}{*}{$\begin{array}{l}\text { Line/ cross } \\
\text { combination }\end{array}$} & \multirow{2}{*}{$\begin{array}{l}\text { Total copy } \\
\text { number of } \\
\text { RF-Oma1 in } \\
\text { zygote }\end{array}$} & \multirow{2}{*}{$\begin{array}{l}\text { Reference } \\
\text { gene }\end{array}$} & \multicolumn{2}{|c|}{ Anther developmental stage } \\
\hline & & & Meiosis & Tetrad \\
\hline \multirow[t]{2}{*}{ TA-33BB-CMS } & 2 & Actin & $0.32 \pm 0.08^{a}$ & $0.17 \pm 0.00$ \\
\hline & & efla & $0.37 \pm 0.06$ & $0.23 \pm 0.01$ \\
\hline \multirow[t]{2}{*}{ TA-33BB-CMS x PI 615522} & 2 & Actin & $0.35 \pm 0.01$ & $0.21 \pm 0.02$ \\
\hline & & efla & $0.35 \pm 0.08$ & $0.30 \pm 0.05$ \\
\hline \multirow[t]{2}{*}{ TA-33BB-CMS x PI 518644} & $3^{b}$ & Actin & $0.59 \pm 0.12$ & $0.37 \pm 0.03$ \\
\hline & & efla & $0.68 \pm 0.08$ & $0.55 \pm 0.15$ \\
\hline
\end{tabular}

${ }^{\mathrm{a}}$ Mean $\pm \mathrm{SD}$

${ }^{b}$ Excluding orf20 $0_{N K-219-1}$ because it is an apparent pseudogene

We constructed FLAG-fused orf $20_{N K-219-1}$, orf $20_{N K-219-2}$ and orf $2 O_{P I} 615522$ controlled by the CaMV $35 \mathrm{~S}$ promoter in binary vectors. Mitochondrial proteins of the transgenic suspension cells were subjected to immunoblot analysis, and neither cell line showed the 200-kDa signal band on BN-PAGE (Fig. 3). In summary, none of the RF-Omal copies identified to date in recessive alleles was found to be $200-\mathrm{kDa}$ generative (summarized in Fig. 1).

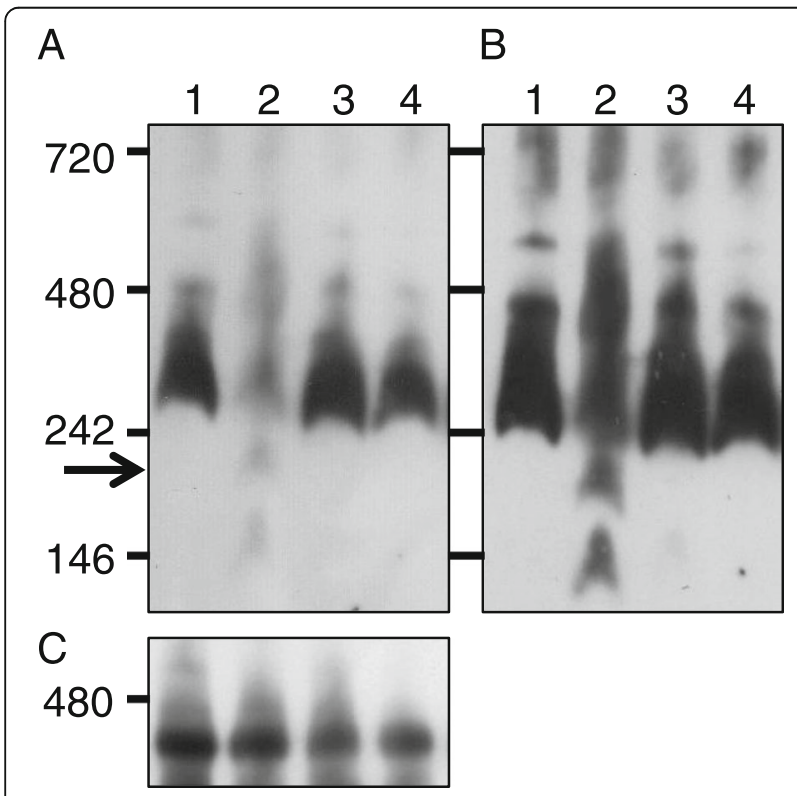

Fig. 4 Immunoblot analysis of total cellular proteins from immature anthers collected from TA-33BB-CMS (lane 1), NK-198 (lane 2), TA33BB-CMS x PI 615522 (lane 3) and TA-33BB-CMS x PI 518644 (lane 4). Protein samples were electrophoresed in Blue Native polyacrylamide gels (4-16\%). Size markers $(\mathrm{kDa})$ are shown on the left. An arrow indicates the 200-kDa band that is the hallmark of post-translational interaction with preSATP6. Immunoblots were probed with anti-preSATP6 (a and $\mathbf{b}$ ) or anti-COXI (c). Exposure times to X-ray film were $10 \mathrm{~s}$ and $1 \mathrm{~min}$ for $\mathbf{a}$ and $\mathbf{b}$, respectively
The 200-kDa generative mRNA quantity can explain the genetic action of the $R f 1$ allele

Having detected the RF-Oma1 mRNA of two recessive alleles and shown that the mRNA quantity appeared to correlate with the copy number of RF-Oma1, counting such $R F-O m a 1$ in the assessment of $R f 1$ strength seemed to be inappropriate. We hypothesized that the genetic action of $R f 1$ alleles is dependent on the sum of the $R F$ Oma1 transcripts that have the potential to generate 200-kDa complexes. To design specific primers to such RF-Oma1 copies, the nucleotide sequences of RF-Oma1 shown in Fig. 1 were aligned to find SNPs or indels specific to forms that can generate $200-\mathrm{kDa}$ complexes (Additional file 4: Figure S2). We found a 3-bp indel that discriminates $200-\mathrm{kDa}$ generative copies from nongenerative ones, and a primer set was designed to specifically amplify the $200-\mathrm{kDa}$ generative $R F$-Oma1. We tested its specificity by using binary vectors each having RF-Oma1 as a template. As shown in Figure S3 (Additional file 5), PCR amplicons of the expected size appeared from 200-kDa generating RF-Oma1 but not from non-generating forms.

Using this primer set, we quantified the mRNA of $R F$ Oma1 in anthers. The homozygotes and heterozygotes of NK-198 $R f 1$ were selected from the $\mathrm{BC}_{6} \mathrm{~F}_{2}$ population. We selected the homozygotes and heterozygotes of NK$305 R f 1$ from a segregating population mentioned in [25]. In this population, NK-305 Rf1 was marked by a specific pattern [p2] of the s17 DNA marker. Total cellular RNA was extracted from anthers at the meiotic and tetrad stages. The results of RT-qPCR are summarized in Table 3. In general, mRNA accumulation was similar between the two developmental stages. The amount of mRNA was highest in the NK-198 Rf1 homozygote, followed by the NK-198 Rf1 heterozygote, the NK-305 $R f 1$ homozygote, and the NK-305 Rf1 heterozygote, in that order. No mRNA was detected from $r f 1 r f 1$ when using this primer set (Table 3 ). 
Table 3 Relative transcript abundance of RF-Oma1 measured by RT-qPCR using common indel characteristics of 200-kDa generative copies $[n=2$ (ef1a of p2p2) or $n=3$ (the others)]

\begin{tabular}{llll}
\hline s17 marker type (genotype) & Reference & \multicolumn{2}{l}{ Anther developmental stage } \\
\cline { 3 - 4 } & gene & Meiosis & Tetrad \\
\hline p1p4 (NK-198 Rf1 heterozygous) & Actin & $0.43 \pm 0.12^{\text {a }}$ & $0.39 \pm 0.02$ \\
& ef1a & $0.48 \pm 0.14$ & $0.48 \pm 0.04$ \\
p1p1 (NK-198 Rf1 homozygous) & Actin & $0.86 \pm 0.21$ & $0.76 \pm 0.18$ \\
& ef1a & $1.00 \pm 0.16$ & $1.05 \pm 0.30$ \\
p2p4 (NK-305 Rf1 heterozygous) & Actin & $0.16 \pm 0.02$ & $0.15 \pm 0.01$ \\
p2p2 (NK-305 Rf1 homozygous) & ef1a & $0.20 \pm 0.01$ & $0.18 \pm 0.06$ \\
& Actin & $0.28 \pm 0.03$ & $0.34 \pm 0.01$ \\
p4p4 (rf1rf1) & ef1a & $0.30 \pm 0.04$ & $0.42 \pm 0.03$ \\
& Actin & Not detected & Not detected \\
\end{tabular}

${ }^{\mathrm{a} M e a n} \pm \mathrm{SD}$

We sought another value that correlated with the allelic strength of $R f 1$. Due to technical difficulties, we were unable to quantify the 200-kDa complex. Instead, we placed our focus on the degree to which the $250-\mathrm{kDa}$ signal intensity was reduced by an $R f 1$ allele (or two $R f 1$ alleles) on BN-PAGE compared with $r f 1 r f 1$ (hereafter we refer to this value as $\left.\Delta^{250 \mathrm{kDa}}\right)$. We had previously shown that the decrease in the amount of the 250-kDa complex is likely caused by an alteration of higher-order structure of preSATP6 because the amount of monomeric preSATP6 is almost unchanged [21], whereas the amount of $420-\mathrm{kDa}$ complex detected by anti-COXI is apparently unaffected [21]. Accumulation of monomeric COXI polypeptide appeared to be comparable between different genotypes [10]. For the NK-198 Rf1, the signal intensity of the $250-\mathrm{kDa}$ signal band in Fig. 2 was normalized with that of the $420-\mathrm{kDa}$ signal band detected by anti-COXI (Table 4$)$. The signal intensity ratio for p1p4 $(0.17 \pm 0.02)$ was reduced by 1.57 compared with $\mathrm{p} 4 \mathrm{p} 4(1.74 \pm 0.26)$, hence the $\Delta^{250 \mathrm{kDa}}$ by a single NK-198 Rf1 was 1.57 . Note that the difference between $\mathrm{p} 4 \mathrm{p} 4$ and $\mathrm{p} 1 \mathrm{p} 1$ was 1.74 , the upper limit of detection by this system.

Arakawa et al. [25] estimated the accumulation of the $250-\mathrm{kDa}$ complex in the homozygotes and heterozygotes of NK-305 $R f 1$ and $r f 1 r f 1$ by the same procedure as our

Table 4 Amount of 250-kDa protein complex in anthers of different genotypes ${ }^{\text {a }}$

\begin{tabular}{lll}
\hline s17 marker type (genotype) & $\begin{array}{l}\mathbf{2 5 0 - k D a} \\
\mathbf{4 2 0 - k D a} \\
\text { ratio }\end{array}$ & $\begin{array}{l}\text { Difference from } \\
\text { rf1rf1 in the same } \\
\left.\text { population } \boldsymbol{\Delta}^{\mathbf{2 5 0 k D a}}\right)\end{array}$ \\
\hline p4p4 (rf1rf1) & $1.74 \pm 0.26$ & - \\
p1p4 (NK-198 Rf1 heterozygous) & $0.17 \pm 0.02$ & 1.57 \\
p1p1 (NK-198 Rf1 homozygous) & Not detected & 1.74 \\
\hline
\end{tabular}

${ }^{a}$ The amount of the $250-\mathrm{kDa}$ complex was estimated by the ratio of the signal intensity between the 250-kDa signal band detected by anti-preSATP 6 and the 420-kDa signal band detected by anti-COXI study. According to [25], the $250-\mathrm{kDa} / 420-\mathrm{kDa}$ ratios were $1.57 \pm 0.16$ and $0.86 \pm 0.07$ for $r f 1 r f 1$ and NK-305 heterozygous, respectively, hence the $\Delta^{250 \mathrm{kDa}}$ by a single NK-305 $R f 1$ copy was 0.71 . The ratio for the NK-305 Rf1 homozygous was $0.12 \pm 0.01$, a value that differed from the rf1rf1 by 1.45 or twice of the $\Delta^{250 \mathrm{kDa}}$ of single NK305 Rf1.

The quantity of mRNA associated with 200-kDa generation and the $\Delta^{250 \mathrm{kDa}}$ were measured in the four genotypes (i.e. homozygous and heterozygous NK-305 Rf1 and NK-198 Rf1). We plotted these data sets as shown in Fig. 5 and Figure S4 (Additional file 6) and found a positive relationship between the level of transcript accumulation and the $\Delta^{250 \mathrm{kDa}}$.

\section{Discussion}

The $250-\mathrm{kDa}$ protein complex containing the CMSspecific polypeptide preSATP6 is the target molecule of $R f 1$ [21]. We were interested in the quantitative aspects of naturally occurring $R f 1$ alleles. The molecular basis for the allelic differences in $R f 1$ likely involves multiple $R F$-Oma1 copies in the allele but not a specific copy.

A notable finding of this study is that all the RF-Oma1 copies in NK-198 Rf1 are capable of generating the 200$\mathrm{kDa}$ complex. This finding was unexpected because transgenics expressing orf $2 O_{N K-198-1}$, orf $2 O_{N K-198-3}$ and orf $20_{N K-198-4}$ were apparently male sterile [23]. Reconciliation between these differing results is possible if a hypomorphic $R f 1$ allele is considered that encodes a single RF-Oma1 capable of generating the $200-\mathrm{kDa}$ complex but barely restores fertility due to the production of a small amount of mRNA [27]. This allele, Fukkokuouba $r f 1$ (see Fig. 1), is non-restoring but plants with this allele sometimes develop anther contents similar to semi-fertile plants (hence, we hesitate to call this allele recessive), as was seen in the three transgenics (our 


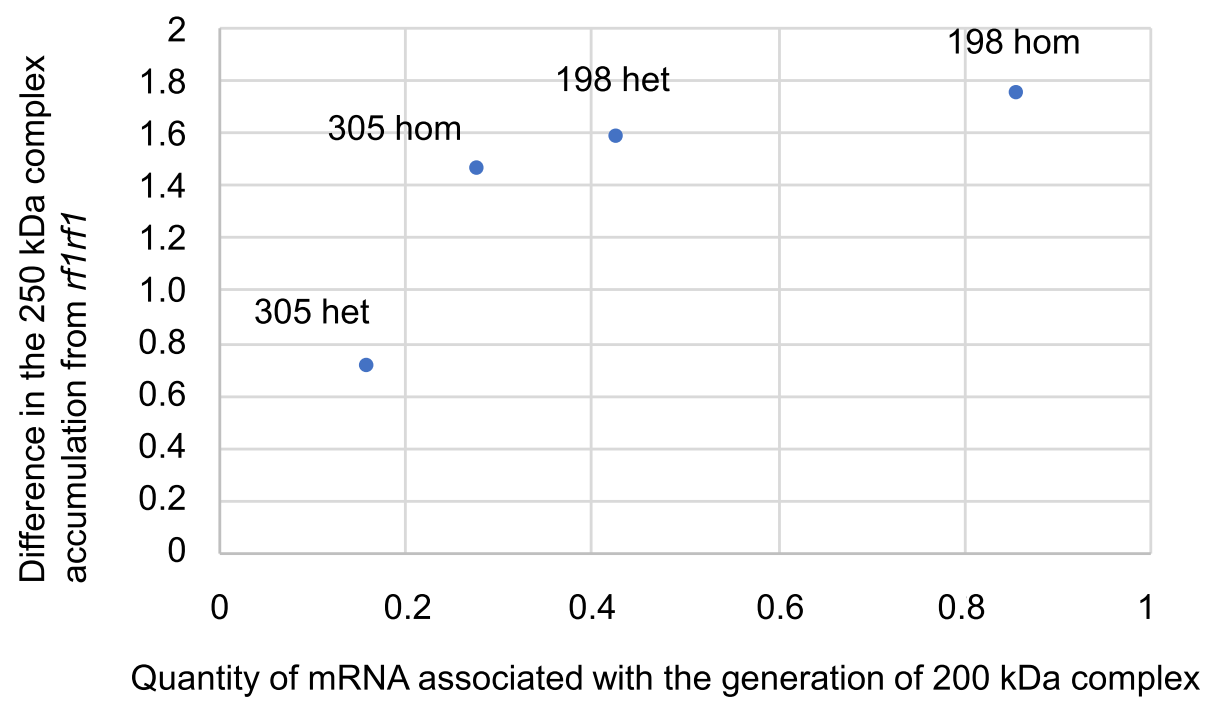

Fig. 5 Relationship between the quantity of mRNA associated with the generation of the 200-kDa complex (horizontal axis) and the relative difference in 250-kDa complex accumulation compared to rf1 ff1 ( $\Delta^{250 k D a}$, vertical axis). Dots represent data from the NK-305 Rf1 heterozygote (305 het), the NK-305 Rf1 homozygote (305 hom), the NK-198 Rf1 heterozygote (198 het), and the NK-198 Rf1 homozygote (198 hom). Anthers were collected at the meiotic stage for RNA isolation, and the reference gene for RT-qPCR was Actin

unpublished observations). According to Arakawa et al. [27] and this study, the amount of orf2O $O_{F u k k o k u}$ mRNA was inferred to be comparable to that in orf $20_{N K-198-1}$, orf $2 O_{N K-198-3}$ or orf $2 O_{N K-198-4}$. It seems possible that each of the three RF-Oma1 copies is functionally equivalent to Fukkoku ouba rf1; hence, transgenics expressing each of these constructs remained male sterile.

We had reported that transgenic sugar beet expressing orf $2 \mathrm{O}_{\mathrm{NK}-198-2}$ was restored to semi-fertility despite the transgene being derived from a strong allele of NK-198 $R f 1$ [23]. This result suggested the possibility that fertility restoration by orf $20_{N K-198-2}$ explains only part of the total strength of NK-198 Rf1. This notion may be supported by the semi-fertile phenotype of the NK-305 Rf1 heterozygote reported in [25], which is very similar to that of the orf $2 O_{N K-198-2}$-expressing transgenics. According to Arakawa et al. [25], NK-305 Rf1 is composed of orf $2 O_{N K-305-1}$ and orf $20_{N K-305-2}$, of which only the former is $200-\mathrm{kDa}$ generative (see Fig. 1). Therefore, data from genotype p2p4 in Table 3 can be interpreted as those coming from orf $20_{N K-305-1}$ mRNA. Based on the figures in Table 1, we estimate that orf $20_{N K-198-2}$ mRNA accounts about $40 \%$ of $\mathrm{p} 1 \mathrm{p} 4$ in Table 3 . Accordingly, our results suggest that orf $2 O_{N K-198-2}$ and orf $2 O_{N K-305-1}$ generate comparable amounts of mRNA. Thus, the phenotypes of transgenic plants described in Matsuhira et al. [23] seem to be consistent with phenotypes expressed by a single NK-305 Rf1 [25]. Altogether, transgenics expressing each of the single dissected RF-Omal copies from NK-198 Rf1 phenocopied the genetic action of other $R f 1$ alleles, but none of them replicated the genetic action of NK-198 Rf1. Of course, we cannot exclude other possibilities such as insufficient expression of the transgene or a background effect are involved in the phenotypes of the transgenics.

Possibly, all four RF-Oma1 in NK-198 Rf1 participate in fertility restoration to achieve completely fertile plants. In this model, the principal restorer is

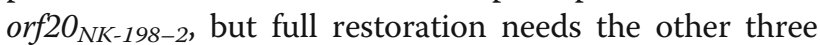
RF-Oma1 copies to provide a sufficient quantity of mRNA to reduce the accumulation of the $250-\mathrm{kDa}$ complex to a level that allows normal pollen development. This model presumes a cumulative effect of RF-Oma1 that can be inferred by the gene dose effect of NK-305 Rf1; its homozygotes were more fertility-restored than the heterozygotes [25]. A gene dose effect on the 250 kDa complex quantity was also obvious in NK-198 Rf1. This cumulative effect is also suggested by Table 3 . We propose that the sugar beet $R f 1$ locus may be a complex locus whose alleles are characterized by the composition of the clustered RF-Oma1 copies.

The strength of the $R f 1$ allele is represented by a reduction in the amount of the $250-\mathrm{kDa}$ complex in anthers (i.e. $\Delta^{250 \mathrm{kDa}}$ ). We estimated the $\Delta^{250 \mathrm{kDa}}$ for a single NK-198 Rf1 as 1.57 (Table 4), which is about twice that of a single NK-305 Rf1 (0.71) (Table 4) and is consistent with the amount of 200-kDa generative mRNA (compare genotypes $\mathrm{p} 1 \mathrm{p} 4$ and $\mathrm{p} 2 \mathrm{p} 4$ in Table 3). Perhaps the amount of the $250-\mathrm{kDa}$ complex is inversely correlated with the quantity of RF-Oma1 mRNA associated with $200-\mathrm{kDa}$ generation. In support of this hypothesis, we found that the amounts of mRNA and the $\Delta^{250 \mathrm{kDa}}$ were 
positively correlated (Fig. 5). This plot poses the notion that NK-198 Rf1 is too efficient when in the homozygous condition as the amount of $200-\mathrm{kDa}$ generative mRNA in the NK-198 Rf1 homozygote is twice that of the heterozygote (Table 3 ), making its potential $\Delta^{250 \mathrm{kDa}}$ equal to $3.14(2 \times 1.57$, see genotype p1p4 in Table 4$)$. This potential $\Delta^{250 \mathrm{kDa}}$, however, cannot be fully directed to the 250-kDa complex because 1.74 (250-kDa accumulation in $r f 1 r f 1$ ) is the upper limit, and the residual 1.40 remains unused. The residual activity would need to be managed if RF-Oma1 has some side-effect that is harmful for the plant, otherwise the frequency of such alleles would decline by counter selection. Although this hypothesis may be related to the observation that the frequency of genotypes that restore full fertility are rare in sugar beet [30], further study is necessary to characterize the genetic diversity of $R f 1$ in sugar beet with the aim of exploiting genetic resources to advance sugar beet breeding.

\section{Conclusions}

Sugar beet $R f 1$ includes alleles of different strengths, including dominant and semi-dominant alleles. The dominant NK-198 Rf1 is composed of four copies of $R F$ Oma1 that have the potential to generate the $200-\mathrm{kDa}$ complex, whereas the semi-dominant NK-305 Rf1 allele has one $200-\mathrm{kDa}$ generative copy and one nongenerative copy. RF-Oma1 copies of recessive alleles have no such activity, but they are transcribed, and the amount of mRNA seems to be copy-number dependent. Using specific primer sets for the $200-\mathrm{kDa}$ generative copies, the mRNA was quantified. The transcript abundance inversely correlated with the quantity of the 250$\mathrm{kDa}$ protein complex composed of preSATP6, the CMSspecific mitochondrial protein. The mRNA quantity also explained the different genetic actions exemplified by NK-198 Rf1 and NK-305 Rf1. We propose a hypothesis in which sugar beet $R f 1$ is a complex locus with multiple alleles whose characters are determined by the function of the RF-Oma1 copies clustered in the alleles. This hypothesis implies that none of the dissected RF-Oma1 copies would be sufficient to restore complete fertility even though they are derived from a strong $R f 1$ allele.

\section{Methods}

\section{Plant materials}

Beet (Beta vulgaris ssp. vulgaris) lines or accessions used or mentioned in this study are listed in Table 5. Sugar beet lines NK-198, NK-219 mm-CMS, NK-305, TA33BB-CMS and TA-33BB-O were developed at the National Agriculture and Food Research Organization, Japan. NK-198 and NK-305 are fertility restored lines [21, 23, 25]. TA-33BB-CMS and TA-33BB-O have identical nuclear genotypes, but the former and the latter
Table 5 Beet lines/accessions used or mentioned in this study

\begin{tabular}{|c|c|c|c|c|}
\hline Line/accession & Cultivar type & Cytoplasm $^{a}$ & Genotype & Origin \\
\hline NK-198 & Sugar beet & $\mathrm{S}$ & Rf1Rf1 & $\mathrm{NARO}^{\mathrm{b}}$ \\
\hline NK-219 mm-CMS & & S & rffrfl & NARO \\
\hline NK-305 & & S & RfiRfi & NARO \\
\hline PI 518644 & & $N$ & rffrfi & NARO \\
\hline PI 615522 & & $N$ & rfirfi & $\mathrm{USDA}^{\mathrm{C}}$ \\
\hline TA-33BB-CMS & & S & rffrfi & USDA \\
\hline TA-33BB-O & & $N$ & RfiRfi & NARO \\
\hline 'Fukkoku ouba' & Leaf beet & $\mathrm{N}$ & rffirfi & NARO \\
\hline
\end{tabular}

${ }^{\mathrm{a} S}$ and $\mathrm{N}$ denote male-sterility inducing cytoplasm and non-male sterility inducing cytoplasm, respectively

${ }^{b}$ National Agriculture and Food Research Organization, Japan

'U. S. Department of Agriculture

have male sterility-inducing and non-inducing mitochondria, respectively. The RF-Oma1 sequences of the two lines are identical to orf $2 O_{T K-81}$ [24]. NK-219 mmCMS is a CMS line competent for Agrobacterium-mediated transformation [31]. PI 518644 and PI 615522 are U.S. sugar beet lines developed by U. S. Department of Agriculture [26]. 'Fukkoku ouba' is a Japanese leaf beet accession [27]. Crosses were done by using paper bags as described in [26]. Plants were grown in the greenhouse or the field at the Field Science Center for the Northern Biosphere, Hokkaido University. Pollen fertility was visually inspected and classified into fully normal, semi-fertile (anthers become orange in color but rarely dehisce), and completely male sterile as described in [25].

\section{Genotyping}

DNA marker s17 was detailed previously [25, 27, 29]. Total cellular DNA was isolated from green leaves by the standard CTAB-based method [32]. The nucleotide sequences of PCR primers are shown in Table S3 (Additional file 7).

\section{Protein complex analysis}

Protein complexes of anthers or crude mitochondria were separated by Blue Native polyacrylamide gel electrophoresis (BN-PAGE) according to [21]. A NativePAGE Novex BisTris Gel system (Thermo Fisher Scientific, Waltham, MA, USA) was used. Separated complexes were blotted onto a Hybond-P PVDF membrane (GE Healthcare, Little Chalfont, UK) according to the manufacturer's instruction manual. Primary antisera were anti-preSATP6 [10], anti-COXI [10], and antiFLAG (Medical and Biological Laboratories, Nagoya, Japan). Antisera were diluted as described in [25]. The secondary antibody was HRP-conjugated goat antimouse IgG and HRP-conjugated goat anti-rabbit IgG 
(Jackson ImmunoResearch, West Grove, PA, USA). Conditions for quantification were modified as previously described [25]. Uncropped images are provided in Additional file 8 .

\section{Transgenic callus}

Similar procedures to those in $[24,25,27]$ were adopted to construct transgenes. Open reading frames (ORFs) of interest were PCR amplified from total cellular DNA as described in [23] and cloned into pDONR/zeo via the Gateway system (Thermo Fisher Scientific). A FLAG tag was fused by in vitro mutagenesis using a PrimeSTAR Mutagenesis Basal Kit (Takara Bio, Kusatsu, Japan). The resultant genes were transferred into $\mathrm{pMDC} \Omega$, a Gateway-compatible binary vector [21]. Transgenes were introduced into NK-219 mm-CMS callus via Agrobacterium LBA 4404 [31]. For the sequences of oligonucleotide primers, see Table S3 (Additional file 7).

\section{Reverse transcription-quantitative PCR}

Anthers from the meiotic or tetrad stages were collected as described in [21]. An RNeasyPlant Mini Kit (Qiagen, Valencia, CA, USA) and RNase-free DNase I (Takara Bio) were used for sample preparation. Complementary DNA was synthesized with SuperScript III First-Strand Synthesis System (Thermo Fisher Scientific) and an oligo $\mathrm{dT}$ primer. Conditions for quantifying transcript levels were followed as described in [25, 27]. Primers for RTqPCR are shown in Table S3 (Additional file 8).

\section{RNA-Seq}

Total cellular RNA was isolated from tetrad stage anthers by using an RNeasy Plant Mini Kit (Qiagen, Valencia, CA, USA). RNA (two $\mu \mathrm{g}$ ) was sent to Macrogen Corp. Japan (Kyoto, Japan) and then quality checked with a 2100 Bioanalyzer (Agilent Technologies, Palo Alto, Calif, USA). The libraries were prepared using a TruSeq Stranded mRNA LT Sample Prep Kit (Illumina, San Diego, CA, USA) and sequenced in pair-ends, 101 bp for each read by Novaseq6000 (Illumina). Raw sequence data were trimmed and quality checked by Sickle ver.1.33 (https://github.com/najoshi/sickle) at a quality threshold of Q20 and a length threshold of $50 \mathrm{bp}$ and FastQC ver.0.11.7 (https://www.bioinformatics.babraham.ac.uk/projects/fastqc/), respectively. Reference sequences are shown in Figure S2 (Additional file 2) and correspond to DDBJ accession numbers AB646133 and AB646135. The filtered reads were aligned to reference sequences using HiSAT2 ver.2.1.0 (https://ccb.jhu.edu/ software/hisat2/index.shtml). The mapped reads having no discrepancy with each reference sequence were visually checked and counted using IGV ver.2.4.13 (https:// software.broadinstitute.org/software/igv/).

\section{Supplementary Information}

Supplementary information accompanies this paper at https://doi.org/10. 1186/s12870-020-02721-9.

Additional file 1: Table S1. Percent identity of amino-acid sequences among RF-Oma1 shown in Fig. 1. Results of pairwise comparison of eleven RF-Oma1 sequences are shown.

Additional file 2: Figure S1. Alignments of reference sequences derived from each RF-Oma1 in NK-198 Rf1. Polymorphic sites of RF-Oma1 in NK-198 Rf1 are shown.

Additional file 3: Table S2 Read-count and ratio of mapped reads on reference sequences. Read-count and ratio of orf2 ${ }_{N K-198-1,}$ orf $20_{N K-198-2,}$ orf2 $_{N K-198-3}$ and orf2 $0_{N K-198-4}$ in anther.

Additional file 4: Figure S2 Alignment of partial nucleotide sequences of RF-Oma1 exon 1. Nucleotide sequences were aligned to design a primer set specific to 200-kDa generative class.

Additional file 5: Figure S3. Agarose gel electrophoresis of PCR products. Specificity of the primer set was tested.

Additional file 6: Figure S4. Scatter plot of quantity of mRNA associated with the generation of 200-kDa complex and difference in the 250-kDa complex accumulation from rf1rf1. Positive correlation between the amount of mRNA of $200 \mathrm{kDa}$ generatives and $\Delta^{250 \mathrm{kDa}}$ in meiosis (actin) and tetrad (actin and efla) stages is shown.

Additional file 7: Table S3 Nucleotide sequences of primers used in this study. Nucleotide sequences of primers used in this study are shown.

Additional file 8. Uncropped images. Uncropped images used for Figs. 2, 3 and 4 are shown.

\section{Abbreviations}

CMS: Cytoplasmic male sterility; PPR: Pentatrico peptide repeat; RFL: Restorerof-fertility like; CaMV: Cauliflower Mosaic Virus; BN-PAGE: Blue-Native polyacrylamide gel electrophoresis; ORF: Open reading frame; RTQPCR: Reverse transcription-quantitative polymerase chain reaction

\section{Acknowledgements}

The authors express their thanks to Sachiyo Ue, Chihiro Sano, and Yohei Kanomata for their excellent technical assistance. Part of this study was conducted at the Field Science Center for the Northern Biosphere, Hokkaido University.

\section{Authors' contributions}

TA and TK designed this study. TA, MM, and KK analyzed transgenic suspension cells with FLAG-fused RF-Oma1. TA quantitatively analyzed the 250-kDa complex and mRNA in anthers with Rf1 alleles. KM analyzed rf1rf1

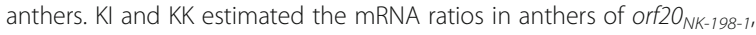
orf20 $0_{N K-198-2}$, orf2 $0_{N K-198-3}$ and orf20 $O_{N K-198-4}$. YK, HM, and TK developed plant materials. TA and KK wrote the draft manuscript. TK supervised the study and finalized the manuscript. All authors read and approved the final manuscript.

\section{Funding}

This work was financially supported in part by JSPS KAKENHI Grant Number 18 K05564 and NARO Bio-oriented Technology Research Advancement Institution (BRAIN) (Research program on development of innovative technology, Grant Number 30001A). TA received JSPS Research Fellowship for Young Scientists (16 J01146). The authors declare that the funding bodies had no role in the design of the study and collection, analysis, and interpretation of data and in writing the manuscript.

\section{Availability of data and materials}

All data generated or analyzed during this study are included in this published article and its supplementary information files. Sequence data were deposited in the DDBJ Sequence Read Archive (DRA010937).

Ethics approval and consent to participate Not applicable. 


\section{Consent for publication}

Not applicable.

\section{Competing interests}

The authors declare that they have no competing interests.

\section{Author details}

${ }^{1}$ Research Faculty of Agriculture, Hokkaido University, Kita-9, Nishi-9, Kita-ku, Sapporo 060-8589, Japan. ${ }^{2}$ Gifu Prefectural Research Institute for Agricultural Technology in Hilly and Mountainous Areas, Nakatsugawa 508-0203, Japan. ${ }^{3}$ Hokkaido Agricultural Research Center, National Agriculture and Food Research Organization, Shinsei Minami 9-4, Memuro 082-0081, Japan.

Received: 25 June 2020 Accepted: 26 October 2020

Published online: 03 November 2020

\section{References}

1. Mackenzie SA. The influence of mitochondrial genetics on crop breeding strategies. In: Janick J, editor. Plant Breeding Reviews. New York: Wiley; 2005. p. 115-38.

2. Chase CD. Cytoplasmic male sterility: a window to the world of plant mitochondrial-nuclear interactions. Trends Genet. 2007;23:81-90.

3. Hu J, Huang W, Huang Q, Qin X, Yu C, Wang L, et al. Mitochondria and cytoplasmic male sterility in plants. Mitochondrion. 2014;19:282-8.

4. Bohra A, Jha UC, Adhimoolam P, Bisht D, Singh NP. Cytoplasmic male sterility (CMS) in hybrid breeding in field crops. Plant Cell Rep. 2016;35:96793.

5. Hanson MR, Bentolila S. Interactions of mitochondrial and nuclear genes that affect male gametophyte development. Plant Cell. 2004;16:S154-69.

6. Budar F, Pelletier G. Male sterility in plants: occurrence, determinism, significance and use. CR Acad Sci Paris Life Sci. 2001;324:543-50.

7. Chen L, Liu Y-G. Male sterility and fertility restoration in crops. Annu Rev Plant Biol. 2014;65:579-606.

8. Rhoads DM, Brunner-Neuenschwander B, Levings CS III, Siedow JN. Crosslinking and disulfide bond formation of introduced cysteine residues suggest a modified model for the tertiary structure of URF13 in the poreforming oligomers. Arch Biochem Biophys. 1998;354:158-64.

9. Duroc Y, Gaillard C, Hiard S, Defrance MC, Pelletier G, Budar F. Biochemical and functional characterization of ORF138, a mitochondrial protein responsible for Ogura cytoplasmic male sterility in Brassiceae. Biochimie. 2005:87:1089-100.

10. Yamamoto MP, Kubo T, Mikami T. The 5'-leader sequence of sugar beet mitochondrial atp6 encodes a novel polypeptide that is characteristic of Owen cytoplasmic male sterility. Mol Genet Genomics. 2005;273:342-9.

11. Schnable PS, Wise RP. The molecular basis of cytoplasmic male sterility and fertility restoration. Trends Plant Sci. 1998;3:175-80

12. Kim Y-J, Zhang D. Molecular control of male fertility for crop hybrid breeding. Trends Plant Sci. 2018:23:53-65.

13. Kubo T, Arakawa T, Honma Y, Kitazaki K. What does the molecular genetics of different types of restorer-of-fertility genes imply? Plants. 2020;9:361.

14. Jaqueth JS, Hou Z, Zheng P, Ren R, Nagel BA, Cutter G, et al. Fertility restoration of maize CMS-C altered by a single amino acid substitution within the Rf4 bHLH transcription factor. Plant J. 2020;101:101-11.

15. Fujji S, Bond CS, Small ID. Selection patterns on restorer-like genes reveal a conflict between nuclear and mitochondrial genomes throughout angiosperm evolution. Proc Natl Acad Sci U S A. 2011;108:1723-8.

16. Kato H, Tezuka K, Feng YY, Kawamoto T, Takahashi H, Mori K, et al. Structura diversity and evolution of the Rf-1 locus in the genus Oryza. Heredity. 2007; 99:516-24.

17. Melonek J, Stone JD, Small I. Evolutionary plasticity of restorer-of-fertility-like proteins in rice. Sci Rep. 2016;6:35152.

18. Melonek J, Zhou R, Bayer PE, Edwards D, Stein N, Small I. High intraspecific diversity of Restorer-of-fertility-like genes in barley. Plant J. 2019;97:281-95.

19. McGrath JM. Panella L: sugar beet breeding. In: Goldman I, editor. Plant Breeding Reviews. New York: Wiley; 2019. p. 167-218.

20. Owen FV. Cytoplasmically inherited male-sterility in sugar beets. J Agr Res. 1945;71:423-40

21. Kitazaki K, Arakawa T, Matsunaga M, Yui-Kurino R, Matsuhira H, Mikami T, et al. Post-translational mechanisms are associated with fertility restoration of cytoplasmic male sterility in sugar beet (Beta vulgaris). Plant J. 2015;83: 290-9.
22. Kaul MLH. Male sterility in higher plants: monographs on Theoretical and Applied Genetics 10. Berlin: Springer-Verlag; 1988.

23. Matsuhira H, Kagami H, Kurata M, Kitazaki K, Matsunaga M, Hamaguchi Y, et al. Unusual and typical features of a novel restorer-of-fertility gene of sugar beet (Beta vulgaris L.). Genetics. 2012;192:1347-58.

24. Arakawa T, Sugaya H, Katsuyama T, Honma Y, Matsui K, Matsuhira H, et al. How did a duplicated gene copy evolve into a restorer-of-fertility gene in a plant? The case of Oma1. R Soc Open Sci. 2019;6:190853.

25. Arakawa T, Ue S, Sano C, Matsunaga M, Kagami H, Yoshida Y, et al. Identification and characterization of a semi-dominant restorer-of-fertility 1 allele in sugar beet (Beta vulgaris). Theor Appl Genet. 2019;132:227-40.

26. Ohgami T, Uchiyama D, Ue S, Yui-Kurino R, Yoshida Y, Kamei Y, et al. Identification of molecular variants of the nonrestoring restorer-of-fertility 1 allele in sugar beet (Beta vulgaris L.). Theor Appl Genet. 2016;129:675-88.

27. Arakawa T, Uchiyama D, Ohgami T, Ohgami R, Murata T, Honma Y, et al. A fertility-restoring genotype of beet (Beta vulgaris $\mathrm{L}$.) is composed of a weak restorer-of-fertility gene and a modifier gene tightly linked to the Rf1 locus. PLoS One. 2018;13:e0198409.

28. Moritani M, Taguchi K, Kitazaki K, Matsuhira H, Katsuyama T, Mikami T, et al. Identification of the predominant nonrestoring allele for Owen -type cytoplasmic male sterility in sugar beet (Beta vulgaris L.): development of molecular markers for the maintainer genotype. Mol Breed. 2013;32:91-100.

29. Taguchi K, Hiyama H, Yui-Kurino R, Muramatsu A, Mikami T, Kubo T. Hybrid breeding skewed the allelic frequencies of molecular variants derived from restorer-of-fertility 1 locus for cytoplasmic male sterility in sugar beet (Beta vulgaris L.). Crop Sci. 2014;54:1407-12.

30. Theurer JC, Ryser GK. Inheritance studies with a pollen fertility restorer sugarbeet inbred. J ASSBT. 1969;15:538-45.

31. Kagami H, Kurata M, Matsuhira H, Taguchi K, Mikami T, Tamagake H, Kubo T. Chapter 27 Sugar Beet (Beta vulgaris L.). In: Wang K, editor. Agrobacterium Protocols, Methods in Molecular Biology, vol. 1223. New York: Springer Science+Business Media; 2015. p. 335-47.

32. Doyle JJ, Doyle JL. Isolation of plant DNA from fresh tissue. Focus. 1990;12: 13-5.

\section{Publisher's Note}

Springer Nature remains neutral with regard to jurisdictional claims in published maps and institutional affiliations.

Ready to submit your research? Choose BMC and benefit from:

- fast, convenient online submission

- thorough peer review by experienced researchers in your field

- rapid publication on acceptance

- support for research data, including large and complex data types

- gold Open Access which fosters wider collaboration and increased citations

- maximum visibility for your research: over $100 \mathrm{M}$ website views per year

At $\mathrm{BMC}$, research is always in progress.

Learn more biomedcentral.com/submissions 